\title{
Época de recolecta en Palo Morado (Peltogyne mexicana M.), en Acapulco Gro., México
}

Time of Gathering in Palo Morado (Mexican Peltogyne M.) in Acapulco Gro.,

Mexico.

\author{
Alejandro Juárez-Agis \\ Universidad Autónoma de Guerrero \\ ajuarezagis@hotmail.com \\ José Ignacio Benítez Villasana \\ Universidad Autónoma de Guerrero \\ bevi_79@hotmail.com \\ Silverio García Sánchez \\ Universidad Autónoma de Guerrero \\ silberio_garcia@hotmail.com
}

\section{Resumen}

En seis arboles de Peltogyne mexicana M. se evaluó el efecto de las fechas sobre variables de germinación, Se utilizó un diseño de bloques al azar con parcelas divididas con cinco bloques (repeticiones), en las parcelas grandes se colocaron los árboles (cinco) y en las parcelas chicas las fechas de recolecta (seis). Cada parcela pequeña tuvo cinco bolsas, estas a su vez tuvieron cinco semillas, usándose 25 semillas de cada fecha de su respectivo árbol (parcela pequeña) en cada parcela grande (fecha de recolecta). Los mejores resultados para germinación fueron para la fecha uno con un valor de $72.48 \%$ y la fecha dos con valor de $61.12 \%$, el valor mínimo fue de 24.48 para la fecha seis. Así las semillas que se recolectan de manera temprana tienen porcentajes de germinación mayor. 
Palabras clave: Peltogyne mexicana, fechas de recolecta, germinación, fechas de recolecta.

\section{Abstract}

Six trees Mexican Peltogyne M. the effect of varying dates on germination was assessed a randomized block design with split plot with five blocks (replicates), on large plots was used trees (five) were placed on the plots and dates girls collects (six). Each plot had five small bags, these in turn had five seeds, 25 seeds of each in use date of its respective shaft (small plot) in each large plot (collection date). The best results for germination were to date one with a value of $72.48 \%$ and date two with value $61.12 \%$, the minimum value was 24.48 for six date. So the seeds are harvested early so have higher germination rates.

Key words: Mexican Peltogyne Dates collects, germination, harvesting dates.

Fecha recepción: Enero 2013

Fecha aceptación: Abril 2013

\section{Introducción}

Peltogyne mexicana Martínez (palo morado) es una especie maderable y es parte de la diversidad florística del estado de Guerrero, por lo que lo sitúa como una de las entidades con mayor diversidad biológica, junto con Oaxaca, Chiapas y Veracruz (CONABIO, 2013). En la actualidad los bosques tropicales son de gran importancia en el mantenimiento de la diversidad y en la regulación del clima global entre otros servicios ambientales, sin embargo, la reducción de los bosques tropicales ha sido ocasionada por factores relacionados con el cambio de uso de suelo lo que ocasiona la fragmentación de los ecosistemas y su disminución a pequeños parches aislados (Wright y Muller-Landau, 2006).

La obtención de conocimientos para el manejo de germoplasma forestal con fines de restauración y reforestación es el inicio del ciclo para el uso de recursos de manera 
sostenible, las semillas o germoplasma pueden ser utilizados con fines comerciales, urbanos, agroforestales o de restauración para resolver problemas económicos, sociales y ambientales, sin embargo, la falta de conocimientos sobre la producción de semillas y tecnología en general para su producción en vivero en muchos casos es nula (FAO, 2006).

El poco conocimiento que se tiene de especies tropicales como fechas de colecta comportamiento de crecimiento en condiciones en vivero o en campo son algunos de los factores determinantes para el éxito de las plantaciones con fines de restauración. Con base en lo anterior conocer la fecha de colecta adecuada esta es el fundamento clave para tener calidad de las semillas ya que influye para el desarrollo y éxito de las plantas. $P$. mexicana M., esta especie se encuentra listada dentro de la Norma Oficial Mexicana NOM 059 SEMARNAT 2010, en la categoría como especie no endémica "A" (amenazada) (Diario Oficial de la Federación 2010).

Un factor determinante en el futuro para la conservación y restauración con palo morado será sembrando semillas con características que aseguren su supervivencia en vivero y campo. Lo cual se logra seleccionando las mejores semillas tomando en cuenta, la fecha de recolecta de las mismas (Montoya, 1996).

Esta especie durante las décadas de los 50-60 fue aprovechada por la belleza de su duramen para la construcción de cascos de navíos y construcciones de tipo residencial aprovechando su dureza y color. De la misma manera esta época coincidió con el auge de la actividad turística del puerto de Acapulco. (Martínez, 1960). Así muchas de las residencias ocuparon esta madera preciosa para la construcción de mesas, vigas y puertas. Esta especie se distribuye en Acapulco en el área de Cumbres de Llano Largo, Las Brisas, y parte de la bahía de Puerto Márquez, Gro; estas son zonas con uso de suelo residencial lo que pone en riesgo la permanencia de esta especie. ( $P$. mexicana $M)$, esta especie representativa y característica de esta zona que actualmente se encuentra en la Norma Oficial Mexicana 059 Secretaría de Medioambiente y Recursos Naturales. (NOM 059 SEMARNAT 2010). Bajo la categoría "A" (amenazada) no endémica. Es por ello que 
generar conocimientos básicos para conocer la producción y germinación de semillas de esta especie, será útil para su conocimiento, posible aprovechamiento, restauración y preservación de esta especie, debido a que se carece o existe poca información sobre el manejo en vivero y fechas óptimas de colecta (Lorenzen, 2009).

Por todo lo anterior, la presente investigación aportará conocimientos sobre el manejo de la semilla en la germinación y crecimiento inicial, asimismo servirá para futuras reforestaciones con palo morado. Ante los escenarios que asechan la permanencia del palo morado esta investigación aportará conocimientos para colecta eficiente de semilla y la producción de plántulas para futuros programas de restauración en esta zona y asegurar la permanencia de esta especie con el fin de asegurar el establecimiento y supervivencia de la especie en campo. 


\section{OBJETIVO GENERAL}

Determinar el efecto de la fecha de recolecta semillas sobre las variables físicas de semillas y germinación en $P$. mexicana $\mathrm{M}$.

\section{OBJETIVOS ESPECÍFICOS}

Evaluar las diferencias en características físicas (ancho, largo, grosor y peso) de las semillas colectadas en las diferentes fechas de recolecta.

Identificar las diferencias en cuando al efecto de fechas de colecta en las variables de germinación.

Verificar si existe correlación entre las fechas de colecta y las variables de características físicas de semillas y germinación.

\section{MATERIALES Y MÉTODOS}

Ubicación del área de estudio

El presente estudio se realizó en el área de Cumbres de Llano Largo Acapulco, Gro., delimitado por las coordenadas geográficas (UTM) al x: 0408900 y: 1860770, al oeste x: 0409029 y: 1860787, al x: 0409054 y: 1860655, al norte x: 049280 y: 1860666. 


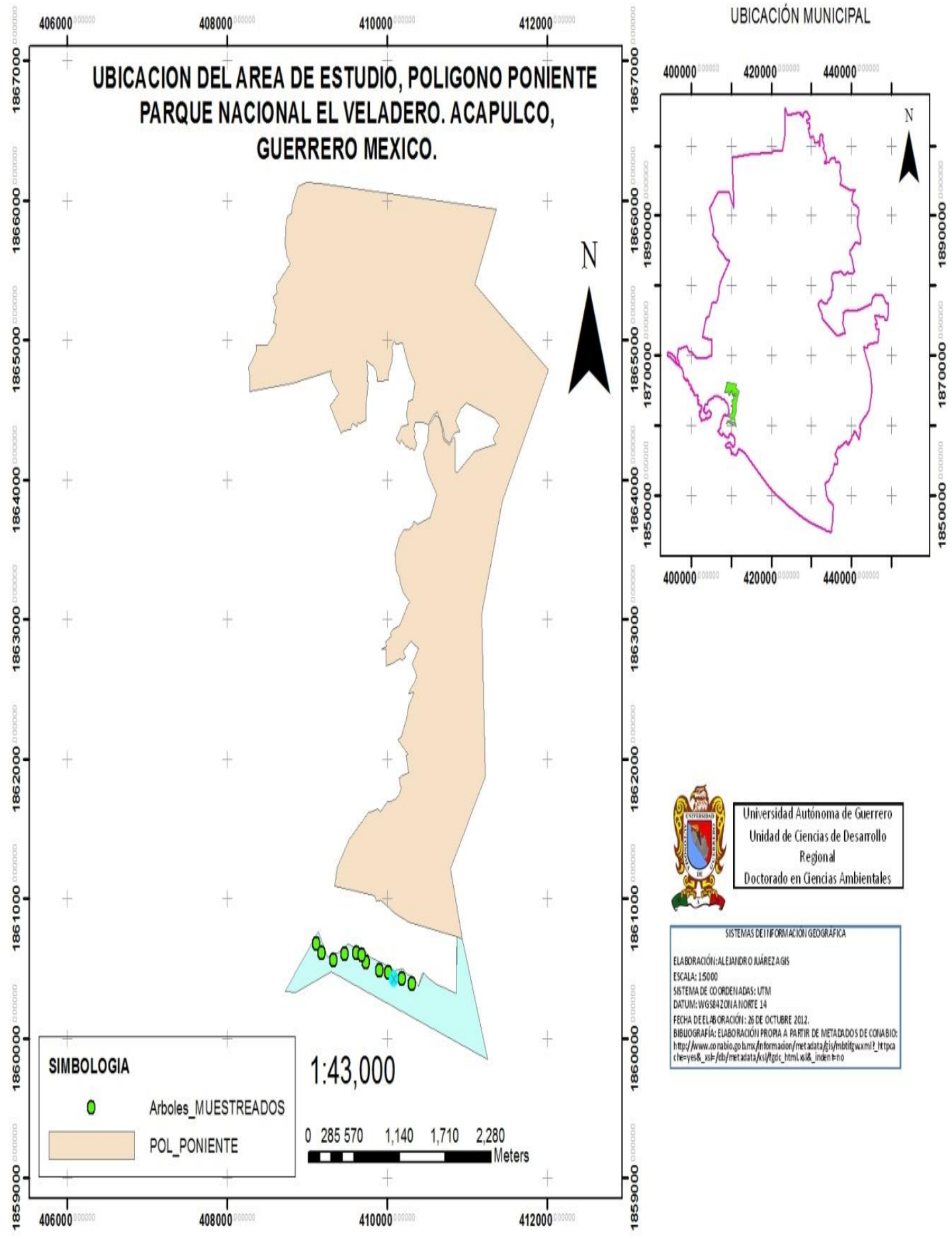

Ubicación del arbolado utilizado en el estudio. 
Muestra

La muestra está conformada por una población de 13 árboles de palo morado ( $P$. mexicana). Los arboles fueron elegidos previo recorrido en el área de estudio, se efectuaron 10 recolectas en diferentes fechas, cabe mencionar que se utilizaron solo cinco fechas, tomando en cuenta que eran las que tenían cantidades de semillas necesarias para las mediciones y siembra, el análisis de características físicas de semilla (peso, ancho, largo y grosor) se realizó contando y midiendo 50 semillas de cada fecha de recolecta.

Fechas de recolecta, número de semillas y arboles muestreados.

\begin{tabular}{|c|c|c|c|c|c|c|}
\hline & \multicolumn{6}{|c|}{ Fechas de recolecta } \\
\hline & 5 & 6 & 7 & 8 & 9 & 10 \\
\hline & $26 / 03 / 2011$ & $02 / 04 / 2011$ & $09 / 04 / 2011$ & $16 / 04 / 2011$ & $23 / 04 / 2011$ & $30 / 04 / 2011$ \\
\hline Árbol 1 & 1323 & 1183 & 933 & 422 & 280 & 146 \\
\hline Árbol 2 & 2440 & 1008 & 553 & 121 & 88 & 56 \\
\hline Árbol 3 & 386. & 562 & $\mathrm{~N} / \mathrm{T}$ & $\mathrm{N} / \mathrm{T}$ & $\mathrm{N} / \mathrm{T}$ & $\mathrm{N} / \mathrm{T}$ \\
\hline Árbol 12 & 1524. & 2242 & 8844 & 344 & 127 & 68 \\
\hline Árbol 13 & 1221. & 1080 & 1732 & 484 & 189 & 67 \\
\hline
\end{tabular}

\section{DISEÑO EXPERIMENTAL}

Se utilizó un diseño de bloques al azar con parcelas divididas con cinco bloques (repeticiones), en las parcelas grandes se colocaron los árboles (cinco) y en las parcelas chicas las fechas de recolecta (seis). Cada parcela pequeña tuvo cinco bolsas, estas a su vez tuvieron cinco semillas, usándose 25 semillas por de cada árbol (parcela pequeña) en cada parcela grande (fecha de recolecta). Así se usaron 750 semillas de cada fecha y en total 3,750 semillas para todo el experimento. 


\section{ANÁLISIS ESTADÍSTICO}

Para verificar diferencias entre fechas de las variables medidas, se utilizó el análisis de varianza y la comparación de medias de Tukey $(\alpha=0.05)$ empleando el procedimiento PROC GLM del programa estadístico SAS versión 9.0 (SAS, 2002). Asimismo, con el coeficiente de correlación de Pearson $(\alpha=0.05)$ se utilizó para calcular las correlaciones de los valores promedio por fecha de recolecta (Juárez et al., 2006) para el largo, ancho, espesor, peso de semilla y también las variables de germinación con la variables fecha de recolección. Para ello se utilizó el procedimiento PROC CORR (SAS, 2002).

Para conocer las diferencias entre las variables de germinación y efecto de árbol y fecha de recolecta se utilizó el siguiente modelo estadístico (Juárez, 2006):

$Y i j=M+A i+F i(j)+A F i j+E i j$

Dónde:

Yij=valor de la observación establecida en el i-ésimo árbol de la j-ésima fecha de recolecta.

$M=$ Efecto de la media general.

$A i=$ efecto del i-ésimo árbol

$\mathrm{Fi}(\mathrm{j})=$ Efecto de la j_ésima fecha de recolecta anidado en el i-ésimo árbol

AFij = efecto de la interacción entre el i-ésimo árbol y la j-ésima fecha de recolecta

Eij = Efecto del error experimental que corresponde al error de la j-ésima fecha de recolecta en el i-ésimo árbol.

El análisis se realizó con paquete $\mathrm{SAS}^{\circledR}$. Con los procedimientos PROC MIXED y otro con GLM (Tukey), para la comparación de medias se verifico la deficiencia entre fechas de recolecta. 
Análisis entre fechas:

$Y i j=M+F i+A i(j)+A F i j+E i j$

Dónde:

Yij= valor de la observación establecida en el i-ésima fecha de recolecta del j-ésimo árbol.

$\mathrm{M}=$ Efecto de la media general.

$\mathrm{Fi}=$ Efecto de la i-ésima fecha de recolecta

$\mathrm{Ai}(\mathrm{j})=$ Efecto del j_ésimo árbol anidado en la i-ésima fecha de recolecta

FAij = efecto de la interacción entre la j-ésima fecha de recolecta y el i-ésimo árbol

Eijk = Efecto del error experimental que corresponde al error de la k-ésima planta en la jésimo árbol de la en el i-ésima fecha de recolecta

Las recolectas se realizaron durante la época de producción de semillas que duró dos meses con total de diez recolectas iniciando el 26 de febrero al 30 de marzo de año 2011. La siembra se realizó el 8 de enero del 2011 y se contaron cada dos días a partir de la siembra para evaluar germinación.

\section{RESULTADOS Y DISCUSIÓN}

Las variables evaluadas para fecha de recolecta fueron largo, ancho, espesor y peso de semilla, presentaron diferencias significativas $(\alpha=0.05)$, siendo la variable peso la que presento mayores diferencias dentro de fecha con un valor de $0.6524 \mathrm{~g}$ para la fecha uno, de manera contraria las variables largo ancho y espesor mostraron pocas diferencias significativas. Observando en general las fechas más tempranas presentan semillas más pesadas que las fechas tardías, así mismo las semillas más largas se presentan en las primeras fechas de colecta. 
Valores promedio y errores estándar para las características físicas de semilla para las 5 fechas de recolecta.

\begin{tabular}{lllll}
\hline Fechas & $\begin{array}{l}\text { Largo } \\
(\mathrm{mm})\end{array}$ & $\begin{array}{l}\text { Ancho } \\
(\mathrm{mm})\end{array}$ & $\begin{array}{l}\text { Espesor } \\
(\mathrm{mm})\end{array}$ & $\begin{array}{l}\text { Peso } \\
(\mathrm{g})\end{array}$ \\
\hline 1 & $1.9780 \pm 0.0057 \mathrm{a}$ & $1.2466 \pm 0.0060 \mathrm{a}$ & $0.3677 \pm 0.0030 \mathrm{a}$ & $0.6524 \pm 0.0048 \mathrm{a}$ \\
\hline 2 & $1.9568 \pm 0.0057 \mathrm{~b}$ & $1.2376 \pm 0.0060 \mathrm{~b}$ & $0.3756 \pm 0.0025 \mathrm{~b}$ & $0.6352 \pm 0.0048 \mathrm{a}$ \\
3 & $1.9776 \pm 0.0067 \mathrm{a}$ & $1.2456 \pm 0.0051 \mathrm{a}$ & $0.3772 \pm 0.0030 \mathrm{~b}$ & $0.6411 \pm 0.0056 \mathrm{a}$ \\
4 & $1.9471 \pm 0.0067 \mathrm{~b}$ & $1.2216 \pm 0.0051 \mathrm{~b}$ & $0.3732 \pm 0.0030 \mathrm{~b}$ & $0.6351 \pm 0.0056 \mathrm{a}$ \\
5 & $1.9427 \pm 0.0067 \mathrm{~b}$ & $1.2211 \pm 0.0060 \mathrm{~b}$ & $0.3722 \pm 0.0030 \mathrm{~b}$ & $0.6281 \pm 0.0056 \mathrm{~b}$ \\
6 & $1.9322 \pm 0.0067 \mathrm{~b}$ & $1.2196 \pm 0.0060 \mathrm{~b}$ & $0.3688 \pm 0.0025 \mathrm{~b}$ & $0.6126 \pm 0.0056 \mathrm{c}$
\end{tabular}

tValores seguidos por distinta literal en la misma columna muestran diferencia significativa (Tukey, $\mathrm{p} \leq 0.05$ ).

Para capacidad germinativa la fecha que presentó mayor porcentaje fue la fecha uno con un valor de $72.48 \%$ y la fecha dos con valor de $61.12 \%$, el valor mínimo fue de 24.48 para la fecha seis. Así las semillas que se recolectan de manera temprana tienen porcentajes de germinación mayor.

La fecha que presentó mayor germinación media diaria fue la fecha correspondiente número dos, con un valor máximo de 4.07 días, presentando un valor mínimo de 1.71 días, la fecha la fecha seis.

Valores promedio y errores estándar para las variables de germinación para las 5 fechas de recolecta.

Fechas Capacidad germinativa Germinación 


\begin{tabular}{lll}
\hline & $(\%)$ & $\begin{array}{l}\text { media diaria } \\
\text { (Días) }\end{array}$ \\
\hline 1 & $72.48 \pm 2.83 \mathrm{~b}$ & $3.14 \pm 0.58 \mathrm{a}$ \\
2 & $61.12 \pm 2.83 \mathrm{~b}$ & $4.07 \pm 0.58 \mathrm{a}$ \\
3 & $45.12 \pm 2.83 \mathrm{a}$ & $1.71 \pm 0.58 \mathrm{~b}$ \\
4 & $44.16 \pm 2.83 \mathrm{~b}$ & $2.03 \pm 0.58 \mathrm{~b}$ \\
5 & $25.76 \pm 2.83 \mathrm{~d}$ & $1.71 \pm 0.58 \mathrm{~b}$ \\
6 & $24.48 \pm 2.83 \mathrm{~d}$ & $2.03 \pm 0.58 \mathrm{~b}$
\end{tabular}

tValores seguidos por distinta literal en la misma columna muestran diferencia significativa (Tukey, $p \leq 0.05$ ).

Dentro del efecto fecha se observan correlaciones significativas para las diferentes variables, una correlación destacada es la que ocurre entre peso de semilla y capacidad germinativa, la cual describe que las semillas más pesadas son las que germinan más, por lo que al relacionarlas con las fechas estas semillas se encuentran en las fechas 1 y 2 del 26 de marzo y 2 de abril.

Otras que resalta, es entre ancho de semilla y capacidad germinativa, en la cual podemos observar que las semillas más anchas son las que tienen mejor capacidad germinativa. 
CORRELACIÓN ENTRE VARIABLES

Coeficientes de correlación de Pearson ( $r$ ) calculados entre variables para las características de semillas y plántulas $(n=5)$.

\begin{tabular}{|c|c|c|c|c|c|c|}
\hline & $\begin{array}{l}\text { peso de } \\
\text { semilla }\end{array}$ & $\begin{array}{l}\text { ancho de } \\
\text { semilla }\end{array}$ & $\begin{array}{l}\text { Largo de } \\
\text { semilla }\end{array}$ & $\begin{array}{l}\text { espesor } \\
\text { de } \\
\text { semilla }\end{array}$ & $\begin{array}{l}\text { Capacidad } \\
\text { germinativa }\end{array}$ & $\begin{array}{l}\text { germinación } \\
\text { media diaria }\end{array}$ \\
\hline $\begin{array}{ll}\text { peso de } \\
\text { semilla }\end{array}$ & 1.00000 & $0.81532^{9}$ & $0.90814^{\pi}$ & 0.12354 & 0.85259 ๆ & 0.35128 \\
\hline $\begin{array}{l}\text { ancho de } \\
\text { semilla }\end{array}$ & & 1.00000 & $0.96118^{\S}$ & 0.22448 & 0.79869 ๆ & 0.44022 \\
\hline $\begin{array}{l}\text { Largo de } \\
\text { semilla }\end{array}$ & & & 1.00000 & 0.24055 & $0.76715^{\text {ๆ }}$ & 0.26796 \\
\hline $\begin{array}{l}\text { espesor de } \\
\text { semilla }\end{array}$ & & & & 1.00000 & -0.00029 & -0.01617 \\
\hline $\begin{array}{l}\text { Capacidad } \\
\text { germinativa }\end{array}$ & & & & & 1.00000 & 0.7526 "2 \\
\hline $\begin{array}{l}\text { germinación } \\
\text { media diaria }\end{array}$ & & & & & & 1.00000 \\
\hline
\end{tabular}

I Significativo $p \leq 0.05$

Con respecto a la variable germinación y tamaño, las semillas que se colectaron en las primeras fechas tienen los porcentajes de germinación más altos; así mismo las variables: capacidad germinativa, germinación media diaria y velocidad germinativa mostraron los resultados más altos también para las primeras fechas. Esto es congruente con los presentado por Puente (1995), donde realiza un experimento donde investiga la fecha de colecta, con el contenido de humedad, así las primeras fechas de recolecta resultaron ser las mejores en cuanto a germinación, todo esto pudiéndose relacionar con el contenido de 
humedad de la semillas, así a medida que pasan los días las semillas van perdiendo humedad y con esto viabilidad, con esto observó que semillas con bajos contenidos de humedad presentaron porcentajes menores de humedad.

De la misma manera Hernández (1995), analizó la humedad de Calophyllum brasilense, Tabebuia guayacan, Terminalia amazonia y encontró que la germinación disminuye conforme los niveles de humedad bajan los porcentajes de germinación en las semillas de estas especies. Con esto la madures fisiológica de las semillas de palo morado se alcanza en las primeras fechas de colecta expresándose en mejores porcentajes de germinación y a medida que la semilla permanece más tiempo en el árbol pierde humedad y viabilidad.

En lo referente al estudio de las semillas, se encontraron diferencias significativas en el tamaño y peso de las mismas para el sitio antes mencionado, resultados que coinciden con los reportados por Plancarte (op. cit.), quien encontró diferencias altamente significativas entre familias del mismo sitio para el peso de semillas.

La variación presente puede ser producto del aislamiento, del tamaño de la población y de la presión de selección, factores importantes en la diferenciación genética de las poblaciones (Wright, 1964).

De la interacción árbol-fecha se observa una tendencia a que las primeras fechas presenten porcentajes más altos de germinación, indicando esto que la vida de los embriones se vea afectada por la permanencia de la semilla en el árbol, así, mientras las semillas que permanecen más tiempo en el árbol están más expuestas a las condiciones ambientales de radiación y temperatura, lo que afecta la viabilidad de la semillas.

Entre la interacción árbol-fecha capacidad germinativa total se puede observar que de la misma manera que las semillas de las primeras fechas son las que sobreviven más pudiéndose relacionar con la permanencia de las mismas en el árbol o también pudiéndose atribuir a características genéticas deseables en los padres. 


\section{Conclusión}

El valor más alto en capacidad germinativa le corresponde a la fecha uno con un valor de $72.48 \%$, y el valor más bajo $24.48 \%$ correspondiente a la fecha seis respectivamente, es recomendable realizar recolectas de semilla para la especie en las primeras fechas ya que se observan los mejores porcentajes de germinación, también, en las primeras fechas se observan valores más altos en las variables de largo $(\mathrm{mm})$, ancho $(\mathrm{mm})$, peso $(\mathrm{gr})$ de semilla.

Se observa que los arboles presentan valores más altos de germinación en las fechas más tempranas de recolecta, disminuyendo porcentajes de germinación conforme la época de producción de semilla avanza. 


\section{Bibliografía}

Gold, K.; P. León-Lobos. \& M. Way. (2004). Manual de recolección de semillas de plantas silvestres para conservación a largo plazo y restauración ecológica. Instituto de Investigaciones Agropecuarias, Centro Regional de Investigación Intihuasi, La Serena.

Jiménez, Q. (1999). Árboles maderables en peligro de extinción en Costa Rica. Instituto Nacional de Biodiversidad.

Juárez-Agis Alejandro, Cuauhtémoc Sáenz-Romero, Jesús Vargas Hernández, Javier López Upton. (2006). Variación geográfica en la germinación y crecimiento inicial de plántulas de Pseudotsuga menziesii de México. Agrociencia 40 (6), 783-792

Martínez, M (1960). Una especie de Peltogyne en México. Anales de instituto de Biología. México, D.F.: UNAM.

Puente C. (1995). Avances en la producción de semillas forestales en América Latina. Memorias de simposio CATIE. 139-143.

ROBLES S., R. (2010). Calidad de planta y variación de semillas en Enterolobium cyclocarpum (jacq.) griseb. En la región costa de Oaxaca. 